\title{
Alterações no leucograma de felinos domésticos (Felis catus) decorrentes de estresse agudo e crônico
}

\author{
Alterations on leukogram of domestic felines (Felis catus) due to \\ acute and chronic stress
}

\author{
Ana Laura Pinto D’Amico Fam ${ }^{[a]}$, Rita Maria Venâncio Mangrich Rocha ${ }^{[b]}$, \\ Cláudia Turra Pimpão ${ }^{[\mathrm{c}]}$, Marúcia de Andrade Cruz ${ }^{[\mathrm{d}]}$
}

[a] Médica veterinária residente em Patologia Clínica, Pontifícia Universidade Católica do Paraná (PUCPR), São José dos Pinhais, PR - Brasil, e-mail: anadamico@gmail.com

[b] Médica veterinária, Mestre, professora de Bioquímica Geral e Aplicada e Patologia Clínica Aplicada, Pontifícia Universidade Católica do Paraná (PUCPR), Curitiba, PR - Brasil. e-mail: rita.rocha@pucpr.br

[c] Médica veterinária, professora Doutora de Farmacologia Geral e Aplicada, Pontifícia Universidade Católica do Paraná (PUCPR), Curitiba, PR - Brasil, e-mail: claudia.pimpao@pucpr.br

[d] Médica veterinária, Curitiba, PR - Brasil, e-mail: maniadegato@maniadegato.com.br

\section{Resumo}

Os hormônios envolvidos com o estresse alteram a quantidade de células brancas presentes no sangue. Durante o estresse agudo, gatos podem desenvolver quadro de linfocitose fisiológica, caracterizado por linfocitose e neutrofilia, graças à liberação de catecolaminas. A linfopenia é verificada quando há liberação de corticosteroides durante o estresse crônico, caracterizando o leucograma de estresse. O presente estudo tem por objetivo avaliar a incidência de leucogramas por estresse na rotina clínica de felinos. Para isso, 93 amostras de sangue de gatos, coletadas em uma clínica especializada, foram analisadas seguindo técnicas padronizadas pelo laboratório. Os resultados foram significativos para os parâmetros Linfócitos Totais, Eosinófilos Totais e Bastonetes ( $\mathrm{p}<0,001)$, sendo que o grupo avaliado apresentou valores menores que a média para os parâmetros Linfócitos e Eosinófilos, e maiores que a média para Bastonetes. Conclui-se que adaptar consultórios para o atendimento de felinos reduz significativamente as alterações sanguíneas decorrentes do medo e da excitação (leucocitose fisiológica). Os valores encontrados, eosinopenia e linfopenia, foram compatíveis com a liberação de glicocorticoide, provavelmente responsiva às doenças e à dor.

Palavras-chave: Felinos. Hemograma. Estresse. Catecolaminas. Glicocorticoides. 


\begin{abstract}
Hormones involved with stress change the amount of white blood cells. During acute stress, cats may develop physiological leykocytosis, characterized by lymphocytosis and neuthophilia due to catecholamine release. Lymphopenia may be verified when there is a corticosteroid release during chronic stress, leading to the stress leukogram. The aim of the present study is to evaluate the incidence of stress leukograms. To carry it out, 93 blood samples from felines, collected in a specialized clinic, were analyzed following standardized techniques by the laboratory. The results were significant for the parameters: total lymphocytes, total eosinophils and band cells $(p<0,001)$, where the evaluated group displayed values lower than the media for the parameter lymphocytes and eosinophils and higher than the media for band cells. It's possible to conclude that clinics programmed to attend felines reduce significantly the blood alterations caused by fear and excitement (physiologic leukocytosis). The found values, eosinopenia and lymphopenia, are compatible with the release of glucocorticoids, probably in response to diseases or pain.
\end{abstract}

Keywords: Felines. Blood count. Stress. Catecholamines. Glucocorticoids.

\title{
Introdução
}

Ao longo dos anos, o ser humano vem mudando a tradição de morar em casas para morar em apartamentos. Esta modificação da estrutura urbana fez com que os gatos fossem cada vez mais solicitados como animais de estimação, visto que não precisam de grandes ambientes para viver, são higiênicos e independentes. Com isso, a população de felinos domésticos vem crescendo. Segundo Almeida, Paixão e Labarthe (2005), estima-se que nos Estados Unidos da América existam aproximadamente 105 milhões de gatos, sendo 75 milhões domésticos e 30 milhões livres. São classificados segundo a forma de criação, como: confinados, mantidos exclusivamente dentro de casa; semiconfinados, mantidos dentro de casa com acesso ao ambiente externo; e livres, aqueles que vivem livremente. No Brasil, com o aumento de pessoas morando em apartamentos, houve um significativo acréscimo de gatos mantidos exclusivamente dentro das residências, visto que não existe o hábito de se levar gatos para passear. Quando esses animais precisam deixar suas casas, por exemplo, para ir ao veterinário, passam por uma situação de medo, excitação ou estresse (DALE, 1990; MEYER et al., 1995; COWELL; DECKER, 2000; SOUZA, 2003). O estresse desencadeia respostas hormonais que alteram a concentração das células sanguíneas (SOUSA, 2007; LEANDRO et al., 2006; ENGLER et al., 2004; DUNN, 2000). Pode ocorrer diminuição da concentração de hemoglobina corpuscular média (CHCM) (WATSON; PAINE, 1943), aumento do número total de eritrócitos (WRIGHT et al., 1951), hematócrito (ERSLEV, 1990), plaquetas (CHATTERJEA et al., 1953) e de leucócitos totais. As células sanguíneas brancas são mais variáveis nos gatos do que nos cães. Isso ocorre porque os felinos possuem um pool marginal muito maior (KOCIBA, 2004; LACERDA, 2005; CLINKENBEARD; MEINKOTH, 2000). Assim sendo, a leucocitose nos gatos pode chegar a valores quatro vezes superiores ao limite de referência (LACERDA, 2005).

A resposta dos animais ao estímulo estressante pode ser dividida em três fases: alteração comportamental, estimulação do sistema simpático (estresse agudo) e ativação do eixo hipotálamo-pituitária-adrenal (estresse crônico) (LANDSBERG, 2006; MALM et al., 2005; SPARKES et al., 1990).

Em situações que causem o estresse agudo, como medo, excitação ou punção venosa, espera-se encontrar um conjunto de alterações, como leucocitose, neutrofilia, eosinofilia e linfocitose, denominado leucocitose fisiológica (KOCIBA, 2004; LATIMER; TVEDTEN, 1999; PRASSE; KAEBERLE; RAMSEY, 1973). Já no estresse crônico, como em casos de dor persistente ou ambientes estressantes, espera-se encontrar o leucograma de estresse, caracterizado por leucocitose, neutrofilia, eosinopenia e linfopenia (YAGI et al., 2004; KERR, 2003; OVERALL, 1997; KLEMM, 1996).

O presente estudo tem por objetivo avaliar hematologicamente 93 gatos, visando a verificar alterações no hemograma de felinos decorrentes de estresse que ocorrem na rotina clínica e verificar diferenças dessa resposta entre animais machos e fêmeas.

Rev. Acad., Ciênc. Agrár. Ambient., Curitiba, v. 8, n. 3, p. 299-306, jul./set. 2010 


\section{Material e métodos}

Os resultados de hemogramas dos gatos atendidos na clínica Mania de Gato durante os meses de janeiro de 2004 e dezembro de 2006 foram tabulados e comparados com os valores de referência existentes para animais normais, fornecidos pelo laboratório onde os exames foram realizados. A clínica Mania de Gato trabalha com agendamento de horários, atendendo prontamente os pacientes que chegam à clínica. Os consultórios são telados, pequenos, com poucos móveis (embutidos), com iluminação indireta e limpos entre as consultas. Foram computados os resultados de 93 hemogramas, sendo 39 de fêmeas e 54 de machos, todos adultos e a maioria sem raça definida. As amostras de sangue foram colhidas em intervalo entre 15 e 30 minutos após a chegada do paciente à clínica, durante a rotina do atendimento, por meio de venopunção da jugular. Os exames foram processados no laboratório habitual utilizado pela clínica, seguindo técnicas padronizadas.

Para a análise estatística dos dados foi utilizado o teste " $t$ " de Student, comparando os valores médios entre machos e fêmeas e entre o grupo todo e os valores de referência. O nível de significância adotado foi de $5 \%(\alpha=0,05)$. Todos os cálculos foram realizados utilizando o software estatístico GraphPad Prism version 3.0 for Windows ${ }^{\circledR}$, San Diego, Califórnia, EUA.

\section{Resultados e discussão}

A Tabela 1 mostra os resultados obtidos para série vermelha. Observam-se valores máximo e mínimo de referência, média das fêmeas, médias dos machos e a média geral de todos os animais.

Tabela 1 - Valores hematológicos fisiológicos (máximos e mínimos), valores médios verificados em gatos domésticos (Felis catus) fêmeas e machos, e a média geral verificada em eritrogramas. Curitiba, PR ( $\mathrm{n}=97)$

\begin{tabular}{lcccccc}
\hline Valores & Idade & $\begin{array}{c}\text { Eritrócitos } \\
(\times \mathbf{1 0} / \boldsymbol{\mu l})\end{array}$ & $\begin{array}{c}\text { Hemoglobina } \\
(\mathbf{g} / \mathbf{d} \mathbf{)})\end{array}$ & $\begin{array}{c}\text { Hematócrito } \\
\mathbf{( \% )}\end{array}$ & VGM & CHGM \\
\hline Valor de referência máximo & & 10 & 15 & 45 & 55 & 36 \\
Valor de referência mínimo & & 5 & 8 & 24 & 39 & 33 \\
Fêmeas ( $\mathrm{n}=40)$ & $7,3 \pm 6,2$ & $7,6 \pm 2,2$ & $11,6 \pm 2,9$ & $36,6 \pm 10,4$ & $48,9 \pm 12$ & $31,8 \pm 5,9$ \\
Machos ( $\mathrm{n}=53)$ & $4,4 \pm 3,6$ & $8,3 \pm 1,8$ & $12,5 \pm 2,8$ & $38,7 \pm 9,2$ & $49 \pm 8,1$ & $31,6 \pm 4,7$ \\
Todos $(\mathrm{n}=93)$ & $5,8 \pm 5,2$ & $7,9 \pm 2$ & $12 \pm 2,9$ & $37,6 \pm 9,7$ & $48,9 \pm 9,9$ & $31,7 \pm 5,2$ \\
\hline
\end{tabular}

Observa-se que nenhum valor possui diferença significativa quando comparado aos valores de referência, assim como não há diferença estatística entre os valores médios das fêmeas e dos machos $(p>0,05)$. $\mathrm{Na}$ série branca houve diferença estatística nos parâmetros Eosinófilos, Linfócitos e Neutrófilos Bastonetes.

A Figura 1 mostra a avaliação do parâmetro Leucócitos Totais, em que se pode observar que não houve diferença estatística entre a média dos animais e a média dos valores de referência.

O parâmetro Linfócitos Totais demonstrou grande diminuição nos animais testados (Figura 2).

Os monócitos (Figura 3) não apresentaram diferença.

A Figura 4 mostra a quantidade de neutrófilos segmentados. Não houve alteração considerável entre as amostras testadas e os valores de referência. Porém, os neutrófilos bastonetes apresentaram elevação significativa, como mostra a Figura 5.

Já a Figura 6 mostra a diferença constatada entre a média dos animais em relação à média dos valores de referência para o parâmetro Eosinófilos. 


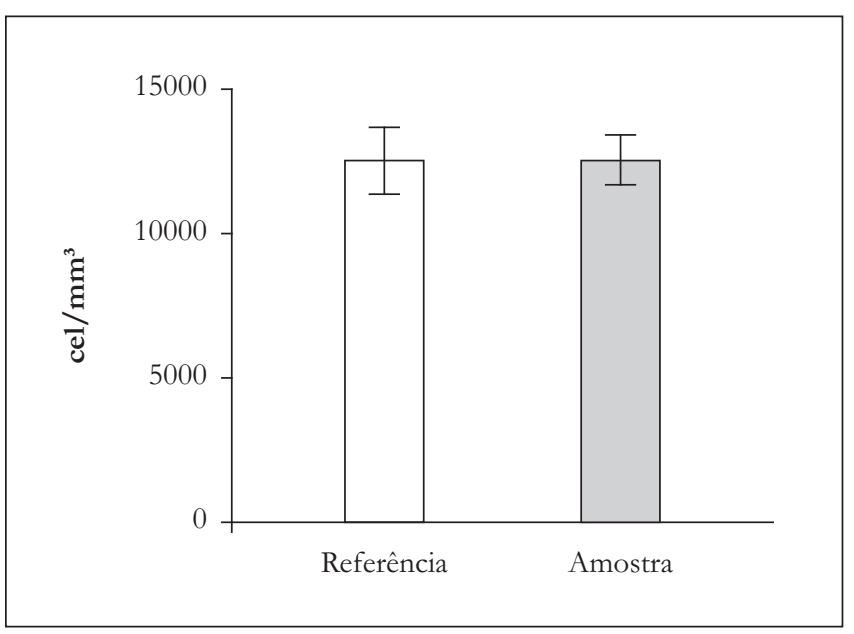

Figura 1 - Comparação do número de leucócitos do sangue $\left(\mathrm{cel} / \mathrm{mm}^{3}\right)$ dos gatos atendidos na clínica Mania de Gato entre os meses de janeiro de 2004 e dezembro de 2006, em relação aos valores normais para a espécie. A abscissa dentro do quadrilátero representa os valores da média; a delimitação do quadrilátero, os percentis de $25 \%$ a $75 \%$; e as ordenadas, o mínimo e o máximo

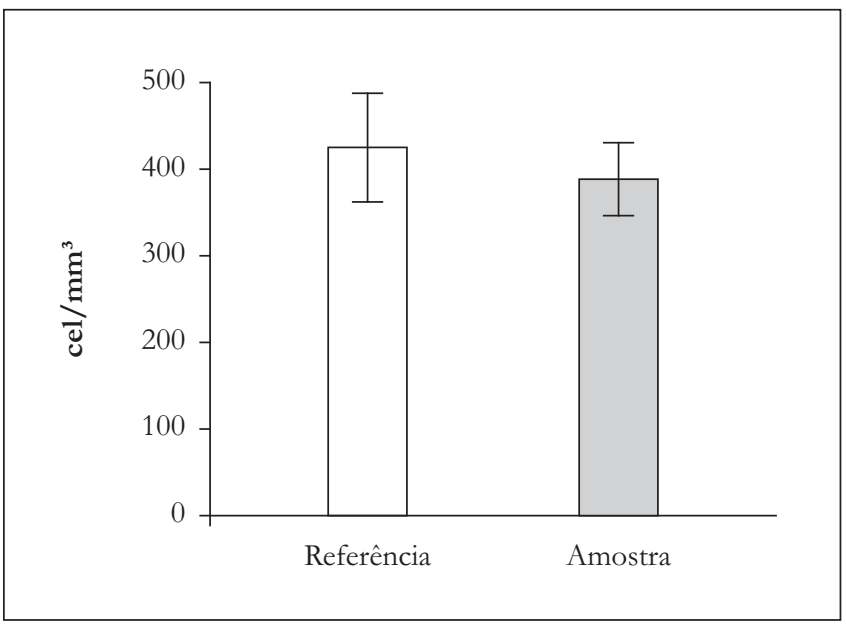

Figura 3 - Comparação do número de monócitos do sangue $\left(\mathrm{cel} / \mathrm{mm}^{3}\right)$ dos gatos atendidos na clínica Mania de Gato entre os meses de janeiro de 2004 e dezembro de 2006 em relação aos valores normais para a espécie. A linha abscissa dentro do quadrilátero representa os valores da média; a delimitação do quadrilátero, os percentis de $25 \%$ a $75 \%$; e as extremidades das barras, ordenadas o mínimo e o máximo

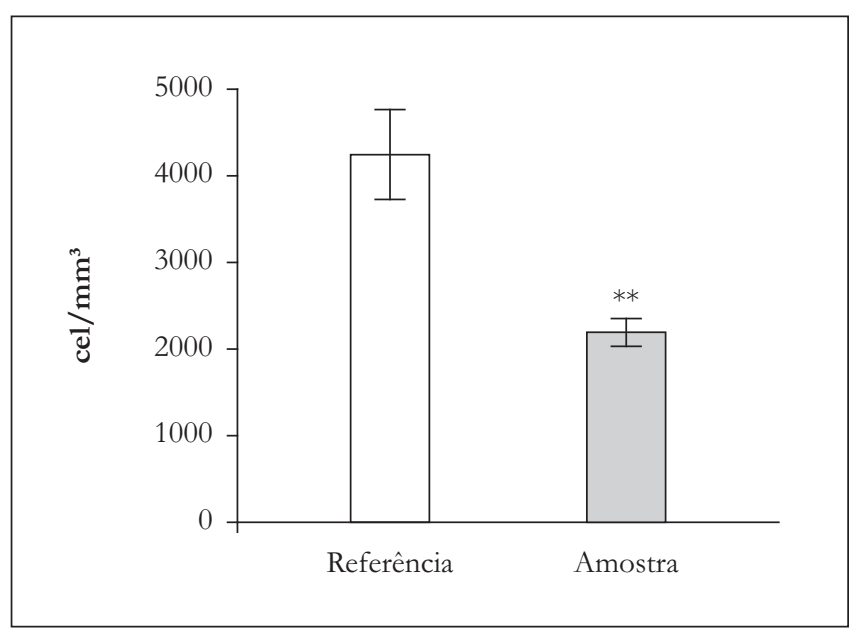

Figura 2 - Comparação do número de linfócitos do sangue $\left(\mathrm{cel} / \mathrm{mm}^{3}\right)$ dos gatos atendidos na clínica Mania de Gato entre os meses de janeiro de 2004 e dezembro de 2006, em relação aos valores normais para a espécie. A abscissa dentro do quadrilátero representa os valores da média; a delimitação do quadrilátero, os percentis de $25 \%$ a $75 \%$; e as extremidades das ordenadas, o mínimo e o máximo

** $\mathrm{p}<0,001$ em relação ao grupo normal

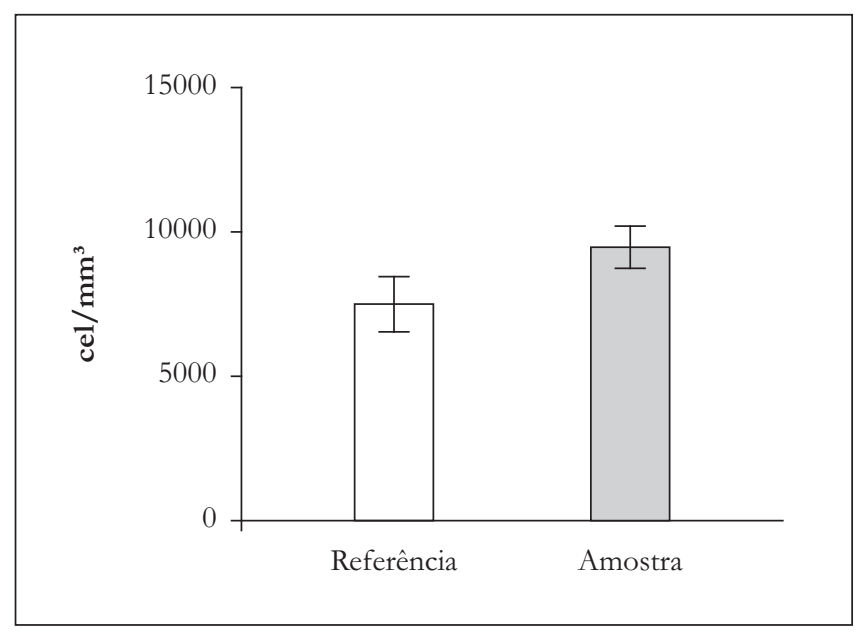

Figura 4 - Comparação do número de neutrófilos segmentados do sangue $\left(\mathrm{cel} / \mathrm{mm}^{3}\right)$ dos gatos atendidos na clínica Mania de Gato entre os meses de janeiro de 2004 e dezembro de 2006, em relação aos valores normais para a espécie. A linha abscissa dentro do quadrilátero representa os valores da média; a delimitação do quadrilátero, os percentis de $25 \%$ a $75 \%$; e as extremidades das barras, ordenadas o mínimo e o máximo 


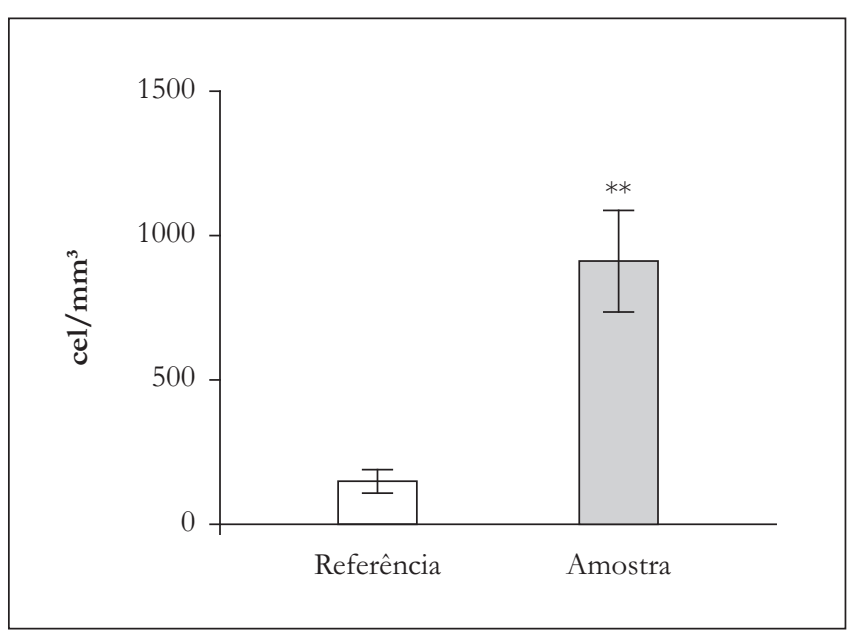

Figura 5 - Comparação do número deneutrófilos bastonetes do sangue $\left(\mathrm{cel} / \mathrm{mm}^{3}\right)$ dos gatos atendidos na clínica Mania de Gato entre os meses de janeiro de 2004 e dezembro de 2006 em relação aos valores normais para a espécie. A linha abscissa dentro do quadrilátero representa os valores da média; a delimitação do quadrilátero, os percentis de $25 \%$ a $75 \%$; e as extremidades das barras, ordenadas o mínimo e o máximo ** $\mathrm{p}<0,001$ em relação ao grupo normal

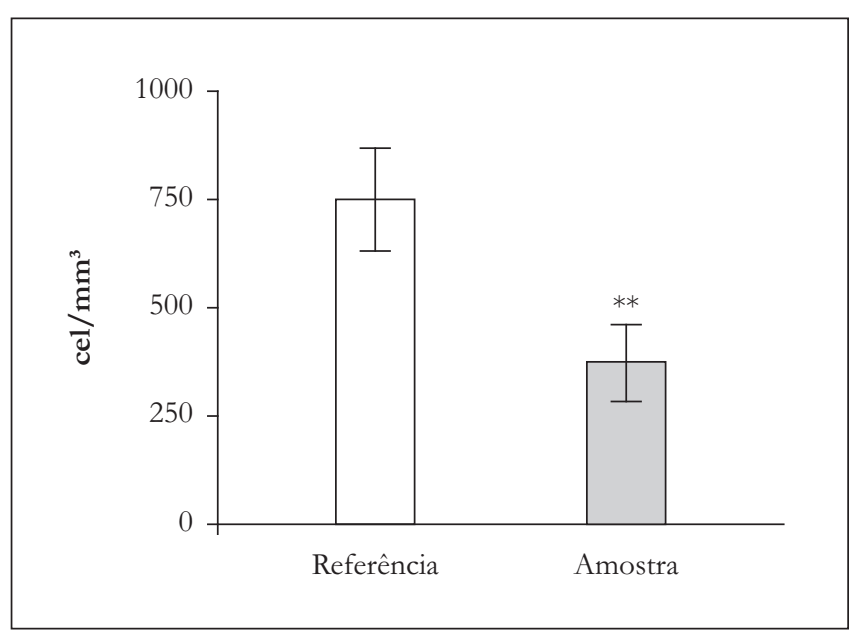

Figura 6 - Comparação do número de eosinófilos $\left(\mathrm{cel} / \mathrm{mm}^{3}\right)$ dos gatos atendidos na clínica Mania de Gato entre os meses de janeiro de 2004 e dezembro de 2006, em relação aos valores normais para a espécie. A linha abscissa dentro do quadrilátero representa os valores da média; a delimitação do quadrilátero, os percentis de $25 \%$ a $75 \%$; e as extremidades das barras ordenadas, o mínimo e o máximo

** $\mathrm{p}<0,001$ em relação ao grupo normal

Segundo Kociba (2004), Latimer e Tvedten (1999), Ishizaki e Kariya (1999), Bounous, Hoskins e Boudreaux (1993) e Prasse, Kaeberle e Ramsey (1973), ambientes estressantes, medo, excitação e punção venosa levam à liberação de adrenalina. Rebar (2003), Nelson e Couto (2001) e Cowell e Decker (2000) apontam que de 20 a 30 minutos após esta liberação ocorrem mudanças no hemograma. Porém, alterações na série vermelha e branca, compatíveis com secreção de adrenalina, não foram evidenciadas após análise das amostras. Isto possivelmente se deve ao fato de que os animais sentem-se mais ambientados nos consultórios da clínica, diminuindo a excitação e o medo e, portanto, a liberação de adrenalina. Outro fator importante é a idade. De acordo com Bounous, Hoskins e Boudreaux (1993) e Cowell e Decker (2000), animais jovens respondem à adrenalina mais intensamente do que adultos. Neste estudo, todos os animais avaliados eram maiores de 1 ano.

Os principais achados durante a avaliação dos resultados foram: eosinopenia e linfopenia. Ambos são característicos do leucograma de estresse. Outras alterações desse quadro incluem leucocitose e neutrofilia, não evidenciadas durante a análise dos dados (SHILOV; ORLOVA, 2000; GARCIA-NAVARRO, 1994; DUNCAN; PRASSE, MAHAFFEY, 1994; JAIN, 1993).

Segundo Greco (2007), a linfopenia é a alteração mais consistente após a liberação de glicocorticoides. Porém, Sousa (2002) acredita que a linfopenia não é um componente marcante no leucograma de estresse de felinos, particularmente. Sendo assim, outras causas poderiam levar à diminuição de linfócitos. Porém, poucas delas levam à diminuição de eosinófilos concomitantemente. Cowell e Decker (2000) afirmam que eosinopenia é difícil ser evidenciada em qualquer espécie. No entanto, é uma das principais alterações encontradas neste estudo.

Sabe-se que o leucograma de estresse leva de quatro a oito horas para aparecer (LACERDA, 2005; REBAR, 2003; WOLF, 1995; ULICH et al., 1988). Como as consultas não demoram esse tempo, outras causas que levam ao aumento de glicocorticoides devem ser lembradas. As principais são doenças e dor (YAGI et al. 2004; KERR, 2003; OVERALL, 1997; KLEMM, 1996; MEYER, 1995). Como o estudo foi realizado em uma 
clínica, alguns dos animais envolvidos nesta pesquisa provavelmente são animais doentes. Este fato também explica por que alto número de neutrófilos bastonetes foi encontrado durante a análise dos resultados, já que muitas doenças levam ao desvio nuclear de neutrófilos à esquerda (DNNE).

Assim como em humanos, não foram evidenciadas diferenças significativas nas respostas hematológicas entre machos e fêmeas (CHATTERJEA; DAMESHEK; STEFANINI, 1953).

\section{Conclusão}

Nas condições do presente estudo, após a avaliação hematológica dos gatos, concluiu-se que adaptar consultórios para o atendimento de felinos reduz significativamente as alterações sanguíneas decorrentes do medo e/ou da excitação (leucocitose fisiológica). Evidenciou-se ainda que não há diferença de resposta entre os sexos. Os valores encontrados, eosinopenia e linfopenia, são compatíveis com a liberação de glicocorticoide, provavelmente responsiva a doenças e à dor.

\section{Referências}

ALMEIDA, F. M.; PAIXÃO, R. L.; LABARTHE, N. V. Superpopulação de gatos domésticos (Felis catus Linnaeus, 1758) urbanos - compreender para controlar. Clínica Veterinária, v. 10, n. 58, p. 44-48, 2005.

BOUNOUS, D. I.; HOSKINS, J. D.; BOUDREAUX, M. K. O sistema hematopoiético. In: HOSKINS, J. D. Pediatria veterinária: cães e gatos até 6 meses de idade. São Paulo: Varela, 1993. p. 317-352.

CHATTERJEA, J. B.; DAMESHEK, W.; STEFANINI, M. The adrenalin (epinephrin) test as applied to hematologic disorders. Journal of the American Society of Hematology, v. 8, p. 211-235, 1953.

CLINKENBEARD, K. D.; MEINKOTH, J. Normal hematology of the cat. In: FELDMAN, B. F.; ZINKL, J. G.; JAIN, N. C. Schalm's veterinary hematology. 5th ed. Baltimore: Lippincott Williams \& Wilkins, 2000. p. 1064-1068.

COWELL, R.; DECKER, L. S. Interpretation of feline leukocyte responses. In: FELDMAN, B. F.; ZINKL, J. G.; JAIN, N. C. Schalm's veterinary hematology. 5th ed. Baltimore: Lippincott Williams \& Wilkins, 2000. p. 382-390.

DALE, D. C. Neutrophilia. In: BEUTTER, E. Williams hematology. 5th ed. New York: McGraw-Hill, 1990. p. 25-37.

DUNN, J. Disorders of leucocyte number. In: DAY, M.; MACKIN, A.; LITTLEWOOD, J. Manual of canine and feline haematology and transfusion medicine. Hampshire: British small animal veterinary association, 2000. p. 245.

DUNCAN, J. R.; PRASSE, K. W.; MAHAFFEY, E. A. Veterinary laboratory medicine: clinical pathology. 3rd ed. Ames: Iowa State University Press, 1994.

ENGLER, H. et al. Effects of social stress on blood leukocyte distribution: the role of alfa and beta-adrenergic mechanisms. Journal of Neuroimmunology, v. 156, p. 153-162, 2004.

ERSLEV, A. J. Secondary polycythemia (erythrocytosis). In: BEUTTER, E. Williams hematology. 5th ed. New York: McGraw-Hill, 1990. p. 709-714.

GARCIA-NAVARRO, C. E. K. Manual de hematologia veterinária. 11. ed. São Paulo: Livraria Varela, 1994.

GRECO, D. S. Avaliação laboratorial das glândulas tireóide, paratireóide, adrenal e pituitária. In: THRALL, M. A. Hematologia e bioquímica clínica veterinária. São Paulo: Roca, 2007. p. 416-430.

ISHIZAKI, H.; KARIYA, Y. Effects of peripheral blood plymorphonuclear leukocyte function and blood components in japanese black steers administered ACTH in a cold environment. Journal of Veterinary Medical Science, v. 61, p. 487-492, 1999. 
JAIN, N. C. Essentials of veterinary hematology. Philadelphia: Lea \& Febiger, 1993.

KERR, M. G. Exames laboratoriais em medicina veterinária: bioquímica clínica e hematologia. 2. ed. São Paulo: Rocca, 2003.

KLEMM, W. R. Fisiologia comportamental. In: SWENSON, M. J.; REECE, W. O. Dukes: fisiologia dos animais domésticos. 11. ed. Rio de Janeiro: Guanabara Koogan, 1996. p. 825-841.

KOCIBA, G. J. Alterações leucocitárias na doença. In: ETTINGER, S. J.; FELDMAN, E.C. Tratado de medicina interna veterinária: doenças do cão e do gato. 5. ed. Rio de Janeiro: Guanabara Koogan, 2004. p. 1941-1956.

LACERDA, L. A. Transfusão sanguínea em veterinária: desafios a vencer. SIMPÓSIO DE PATOLOGIA CLÍNICA VETERINÁRIA DA REGIÃO SUL DO BRASIL, 2., 2005, Porto Alegre. Anais... Porto Alegre: Ed. da UFRGS, 2005. p. 62-81. Disponível em: <http://www.lume.ufrgs.br/bitstream/handle/10183/17307/000456119.pdf?sequence=1>. Acesso em: 13 ago. 2010

LANDSBERG, G. Medical health and behavioral: differentiating neurological and dermatological from behavioral. 2006. Disponível em: < http://www.michvma.org/documents/MVC\%20Proceedings/Landsberg3.pdf>. Acesso em: 13 ago. 2007.

LATIMER, K. S.; TVEDTEN, H. Leukocyte disorders. In: WILLARD, M. D.; TVEDTEN, H.; TURNWALD, G. Small animal clinical diagnosis by laboratory methods. 3rd ed. Pensylvania: Saunders Company, 1999. p. 63-91.

LEANDRO, C. G. et al. Efeito da L-glutamina sobre o perfil leucocitário e a função fagocítica de macrófagos de ratos estressados. Revista de Nutrição, v. 19, n. 4, p. 437-444, 2006.

MALM, C. et al. Ovário-histerectomia: estudo experimental comparativo entre as abordagens laparoscópica e aberta na espécie canina - III: estresse pela análise do cortisol plasmático. Arquivo Brasileiro de Medicina Veterinária e Zootecnia, v. 57, n. 5, p. 584-590, 2005.

MEYER, D. J.; COLES, E. H.; RICH, L. J. Medicina de laboratório veterinária: interpretação e diagnóstico. São Paulo: Roca, 1995.

NELSON, R. W.; COUTO, C. G. Medicina interna de pequenos animais. 2. ed. Rio de Janeiro: Guanabara Koogan, 2001.

OVERALL, K. L. Clinical behavioral medicine for small animals. St Louis: Mosby, 1997.

PRASSE, K. W.; KAEBERLE, M. L.; RAMSEY, F. K. Blood neutrophilic granulocyte kinetics in cats. American Journal of Veterinary Research, v. 34, n. 8, p. 1021-1025, 1973.

REBAR, A. H. Guia de hematologia para cães e gatos. São Paulo: Roca, 2003.

SHILOV, Y. I.; ORLOVA, E. G. Adrenergic regulation of phagocytic activity of peripheral blood neutrophils, monocytes, and eosinophils in stressed rats. Bulletin of Experimental Biology and Medicine, v. 129, n. 5, p. 563-566, 2000.

SOUSA, C. A. The use of corticosteroids in veterinary dermatology. 2002. Disponível em: <http://www.utskinvet. org/pdf/Corticosteroids.pdf>. Acesso em: 13 ago. 2007.

SOUZA, H. J. M. Coletâneas em medicina e cirurgia felina. Rio de Janeiro: L. F. Livros, 2003.

SPARKES, A. H. et al. Assessment of adrenal function in cats: response to intravenous synthetic ACTH. Journal of Small Animal Practice, v. 31, p. 1-4, 1990.

ULICH, T. R. et al. The contributions of adrenal hormones, hemodynamic factors, and the endotoxin-related stress reaction to stable prostaglandin analog-induced peripheral lymphopenia and neutrophilia. Journal of Leukocyte Biology, v. 43, p. 5-10, 1988.

WATSON, C. J.; PAINE, J. R. A study of the splenic venous blood. American Journal of the Medical Sciences, v. 205, n. 4, p. 493-501, 1943. 
WOLF, A. Manual de medicina felina. Zaragoza: Acribia, 1995.

WRIGHT, C. S. et al. Direct splenic arterial and venous blood studies in the hypersplenic syndromes before and after epinephrine. Journal of the American Society of Hematology, v. 6, p. 195-212, 1951.

YAGI, Y. et al. Transport stress increases somatic cell counts in milk, and enhances the migration capacity of peripheral blood neutrophils of dairy cows. Journal of Veterinary Medical Science, v. 66, p. 381-387, 2004.

Recebido: 05/05/2009

Received: 05/05/2009

Aprovado: 03/02/2010

Approved: 02/03/2010 